\title{
Setting up a Finnish NGI Site - Site Perspective
}

\author{
Vera HANSPER* \\ CSC - IT Center for Science \\ P.O.Box 405 \\ 02101 Espoo, Finland \\ E-mail: vera.hansper@csc.fil \\ Kalle Happonen \\ CSC - IT Center for Science \\ P.O.Box 405 \\ 02101 Espoo, Finland \\ E-mail: kalle.happonen@csc.fi

\section{Juha Herrala ${ }^{\dagger}$} \\ Tampere University of Technology \\ PO Box 692, Tampere, Finland \\ E-mail: juha.herrala@tut.fi
}

In 2011, the Finnish Academy granted a sizeable amount of funding to enhance the national computing infrastructure based on grid technologies. The new computing infrastructure is known as the Finnish Grid Infrastructure (FGI), and it represents the Finnish NGI infrastructure towards EGI. Nine Finnish universities and CSC - IT Center for Science - form the consortium that host the sites of the national grid. This work presents (and introduces) both the building of the FGI and the perspective of one of the host partners, Tampere University of Technology, of the procurement and deployment process.

EGI Community Forum 2012 / EMI Second Technical Conference 26-30 March, 2012

Munich, Germany

\footnotetext{
* Speaker.

${ }^{\dagger}$ Presenter at the conference.
} 


\section{Introduction}

The Finnish Grid Infrastructure, FGI, is the national distributed computing environment in Finland. It replaced the previous grid infrastructure, the national Material Science grid (M-Grid) which was established in late 2004. FGI represents the Finnish NGI infrastructure towards EGI. This national infrastructure is comprised of ten sites, nine of which are hosted by major Finnish universities, and CSC, IT Center for Science, as the administrative centre. Tampere University of Technology, highlighted in this paper, is one of the participating universities.

\section{Evolution of Grid Technologies in Finland}

Interest in grid technologies in Finland was in step with the evolution of the need for computer grid systems at the Large Hadron Collider in CERN. Application for funding for an new infrastructure in 2003 was led by Helsinki University and CSC and resulted in the formation of a consortium for procuring Finland's first national materials science grid, known as M-Grid. The M-Grid consisted of semi-heterogeneous configuration at 9 sites across Finnish Universities, Institutes and CSC and was fully operational in 2005. The Advanced Resource Connector (ARC) middleware was chosen for this system and it was loosely connected with NorduGrid through Finland's participation in the WLCG and NDGF-T1. By 2009 it was clear that the infrastructure was ageing and limited with respect to advances in technology. Since by experience with M-grid it had been shown that a collective funding application provides a better outcome for hardware acquisition applications for funding were made with a revised consortium, led by Helsinki University and CSC. The Academy of Finland, under the Ministry of Education and Culture, is the prime funding agency for basic research in Finland and granted 1.4 M euro joint funding at the end of 2010 for 2011. The procurement process was made in 2011 and the new Finnish grid - FGI - began operations in 2012.

\section{FGI Organisation}

The Finnish Grid Infrastructure consortium consists of Aalto University, the University of Helsinki, Lappeenranta University of Technology, Tampere University of Technology, the University of Eastern Finland, the University of Jyväskylä, the University of Oulu, the University of Turku, Åbo Akademi University and CSC - IT Center for Science. Members host the clusters and CSC coordinates the FGI activity by supporting site administrators and providing the software stack, including the grid middleware. The site University administrators are responsible for clusters' day to day administration and local user management. All members collaborate with their security departments and CSC collaborates with all University security departments.

\subsection{The FGI consortium and governance}

The FGI consortium created the FGI board for all members that were part of the funding application. The board was also strongly involved in the procurement of the hardware for the FGI. During the procurement process the board members were instrumental in determining the hardware requirements and the bidding process. In the operational phase of the FGI, each consortium member maintains a presence on the FGI board. The board's function is to determine resource 
allocation, policies and similar high level administrative decisions. They meet regularly twice a year, with further correspondence via email or phone conferences as needed. At the site level, CSC conducts weekly chat meetings for all site administrators to discuss general issues affecting sites as well as developments in software and tools. Sites also provide status reports, and are asked to explain any outstanding issues. Administrators also have face-to-face meetings 3-4 times a year

\section{FGI - putting the pieces together}

\subsection{Procuring a national infrastructure}

Procuring hardware for a large scale infrastructure is an extensive process. According to Finnish and European legislation, an outright purchase by institutions with any one vendor is not permissible and a call for tender must be set up. CSC has had extensive experience in tendering, and therefore led this process. Together with the consortium, decisions on hardware types, together with the expected performance where included in the tender. There was also an issue of time frames, as the funding for the infrastructure had to be used in 2011 - so vendors were given tight time lines for providing offers, and the consortium also had tight time frames for making decisions. The final outcome of the tendering was that Hewlett Packard (HP) was the successful vendor. Actually obtaining a vendor does not end the procurement process. The tender itself was written so that there was a maximum amount of flexibility in the type of system that could be purchased for FGI. This enabled each member to tailor the hardware to their requirements. The FGI consortium worked together with HP in this stage of the process. It was decided that a reasonably homogeneous system would be purchased, though with flexibility in the amount of hardware each site purchased. The final orders were made in Q3 of 2011, with a rather tight deadline to have the hardware delivered, installed and accepted (through various tests) by the end of 2011!

\subsection{FGI hardware}

As sites provided a minimum of 30involvement, through the FGI board and through their technical representatives, in deciding the specific hardware that would be installed locally at their site. The basic hardware configuration consisted of:

\section{Main compute nodes}

HP SL390s G7 Scaleout servers

$2 *$ Intel 6-core Xeon X5650 CPUs

24-96 GB memory (2GB-8GB/core)

$1 T B$ memory fat nodes

\section{GPGPU nodes}

Dual Tesla 2050 or Tesla 2090

\section{Disk servers}

The total disk capacity: 1PB

\section{Infiniband QDR interconnect}

\section{Frontends}

Admin node, Grid node, based on the main compute nodes 
Sites had the freedom to choose from the above list. The size of the clusters at each site varied from 8 to 200 nodes, with a total of around 6000 compute cores and 90 GPGPUs. The overall theoretical peak computing power was calculated to be 154 Tflops, although $2 / 3$ of that amount is actually due solely to the GPGPUs. This is a significant increase on the computing power of the previous M-grid, which had been calculated at 2.5 Tflops.

\subsection{FGI Software}

As FGI represents the infrastructure of the Finnish NGI towards EGI, it was decided that all clusters would run an EGI approved distribution: Scientific Linux 6. The system is set up so that compute nodes are installed via Kickstart though there are also a few homebrew management scripts. SLURM was chosen as the local scheduler and ARC as the grid middleware. CSC also maintains a runtime environment registry for scientific software that can be used both locally and through the grid. CSC also encourages site administrators to participate in contributing to the runtime environment.

\section{An operational infrastructure: Using the FGI}

Continuing from the success of the previous M-Grid, both local cluster from within the host university and nation wide grid use are permitted. This enables users to run applications in the environment that best suits their needs. Grid use is, however, encouraged as much as possible. To ease uptake of grid usage a Finnish catch-all VO for grid use. Other VOs can be supported by sites on a need basis, and although the NGI manager may recommend other VOs it is ultimately up to the sites can decide for themselves whether a VO is supported. It was deliberately decided that the FGI is primarily for non-WLCG use.

\section{Tampere University of Technology (TUT) - a site perspective}

\subsection{Tampere}

Tampere is the third largest town in Finland with over 200,000 inhabitants. It is one of Finland's fastest growing urban centres and, according to a recent survey, the most popular city to live and study in. Tampere hosts two universities: Tampere University of Technology and the University of Tampere, which together have over 25,000 students.

\subsection{TUT - an International University}

TUT was Established in 1965 and has about 10,400 students and 2,000 employees. There are almost 300 foreign nationals among employees and close to 1000 exchange and visiting students annually, with 140 students enrolled in the international MSc programmes in 2010. TUT has established collaborations with 200 universities world wide.

\subsection{Scientific Computing at TUT}

Scientific Computing is offered as a service for computational scientists and research teams within the university. The local computing resources includes a Linux cluster environment with 
$1000 \mathrm{CPU}$ cores + GPUs and a desktop grid environment for distributed computing with 500 computers and $1000 \mathrm{CPU}$ cores. There is also software support and general support for users. Researchers also have access to CSC-maintained computing facilities for larger computing tasks.

\subsection{Site Experiences - General}

The Linux cluster at TUT has been extended from approximately $300 \mathrm{CPU}$ cores to $1000 \mathrm{CPU}$ cores, and storage has increased to over $200 \mathrm{~TB}$ of raw disk space as the financial support of the Academy of Finland allowed TUT to purchase the new computational resources with an affordable own/in-kind funding. The joint tendering process with CSC and other universities resulted into a more lucrative price/performance ratio than what would have been possible if TUT acted as a single entity.

\subsection{Site Experiences - Hardware process}

As CSC shared their extensive legal and technical knowledge with other participating parties, it meant savings of TUT's own resources, and we could still rely on a professional end result of the tendering process. Because of unforeseen circumstances, the overall hardware process from the tendering until the delivery took considerably longer than originally expected. However, this was also because the joint order for the procurement was around ten times larger than TUT's order alone.

\subsection{Site Experiences - Software}

All sites, including TUT, benefit from CSC's role as the software coordinator. They maintain the cluster software environment, including typical scientific software used by the researchers, thus saving the effort required to do this at a site level.

As the operating system, Scientific Linux, had not been in use at TUT before some learning was required to adapt existing codes to the new distribution. Also, the local job submission system was changed from SGE (M-Grid) to SLURM (FGI). This meant some users whose job submission scripts were based on SGE commands needed to make changes.

\subsection{Site Experiences - Grid}

As the actual grid middleware had not changed (the original M-Grid has used NorduGrid ARC since 2004) the changes did not affect the overall Grid experience. It helps that the user documentation is also be based on the ARC user interface. A new development is the integration with EGI, which has meant that the FGI sites and responsible people need to learn the EGI processes and tools. The sites are currently moving to more harmonious deployment, and TUT, as with the other sites, also takes a role in the FGI operations.

\section{References}

[1] CSC - IT Center for Science http://www.csc.fi/english

[2] Finnish Grid Infrastructure

http://www.csc.fi/english/research/Computing_services/grid_environments/fgi 
[3] FGI https://confluence.csc.fi/display/fgi

[4] Scientific Computing at TUT http://www.tut.fi/tesc

[5] Academy of Finland http://www.aka.fi/eng

[6] Scientific Linux http://www.scientificlinux.org/

[7] ARC http://www.nordugrid.org/arc/

[8] EGI http://www.egi.eu/

[9] LHC http://www.cern.ch/

[10] WLCG http://wlcg.web.cern.ch/

[11] NDGF-T1 http://www.ndgf.org/ndgfweb/home.html

[12] HP http://www.hp.com/ 